\title{
Invarianza por sexo y distribución del Índice de Satisfacción Sexual reducido en una muestra mexicana de rutas aleatorias
}

\author{
Invariance according to sex and distribution of low Sexual \\ Satisfaction Index based on Mexican people random route \\ sample
} \section{PPSicogeterte}
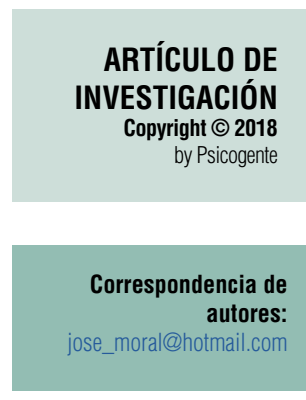

Recibido: 23-03-17 Aceptado: 26-08-17 Publicado: 01-07-18

Cómo citar este artículo (APA):

Moral de la Rubia, J. (2018). Invarianza por sexo y distribución del Índice de Satisfacción Sexual reducido en una muestra mexicana de rutas aleatorias.

Psicogente 21(40), 337-359. https://doi.org/10.17081/psico.21.40.2705

José Moral de la Rubia iD

Universidad Autónoma de Nuevo León, Monterrey, México

Resumen

Objetivo: El Índice de Satisfacción Sexual de Hudson constituye una medición unidimensional de la satisfacción sexual empleada en investigación y clínica. En México, se ha propuesto una forma reducida a 10 ítems del ISS (ISS-10), que ha servido de base a este estudio para aportar nuevas evidencias de validez cruzada (entre mujeres y hombres) y convergente; describir su distribución y estudiar su relación con seis variables sociodemográficas.

Método: Se realizó una investigación instrumental en la que participaron 807 personas casadas o en unión libre de Monterrey, México, a las cuales se les aplicó el ISS-10, la Escala de Valoración de la Relación (RAS), la Escala de Engrandecimiento Marital (MAS) y otras cuatro escalas entre enero y mayo de 2014, mediante un muestreo de rutas aleatorias. Se aplicó análisis factorial confirmatorio multigrupo.

Resultados: El modelo de un factor con 10 indicadores mostró invarianza entre mujeres y hombres, pero las puntuaciones del ISS-10 no siguieron una distribución normal. Además, en la muestra total, la correlación del ISS-10 con la satisfacción con la relación (RAS) fue muy alta $\left(r_{s}=.71\right)$ y con el engrandecimiento marital (MAS), alta $\left(r_{s}=.56\right)$. La edad, los años de casados o en unión libre y la escolaridad presentaron correlaciones bajas con el ISS-10 ( $r_{s}$ de .13 a .27). El sexo y el estado civil fueron independientes del ISS-10.

Conclusión: El Índice de Satisfacción Sexual, como una medición unidimensional de la satisfacción sexual reducida a 10 indicadores, presenta evidencias de validez; independientemente de que se aplique a hombre o mujer, y puede ser baremado a partir de puntuaciones de percentil.

Palabras clave: satisfacción sexual, satisfacción con la relación, engrandecimiento marital, validez de las pruebas, análisis factorial, méxico.

\section{Abstract}

Objective: Hudson's Index of Sexual Satisfaction is a one-dimensional sexual satisfaction measure, used for research and clinical practice. In Mexico, 10 items form (ISS-10) has been proposed. This research aims to provide new evidences related to cross-validity (among women and men) and convergent validity of ISS-10 form, describing distribution, and study and also its relationship with six socio-demographic variables.

Method: An instrumental research was performed. ISS-10, Relationship Rating Scale (RAS), Marital Aggrandizement Scale (MAS) and three other scales were applied; 807 married or cohabiting people were randomly sampled from Monterrey, Mexico between January and May 2014. Multi-confirmatory factorial group analysis was applied for data analysis.

Results: The factor model has 10 indicators that show invariance among women and men. ISS-10 scores did not follow a normal distribution. In the overall sample, ISS-10 correlated with relationship satisfaction (RAS) was very high $\left(r_{s}=.71\right)$, and with the marital aggrandizement (MAS) also was high $\left(r_{s}=.56\right)$. Age, years of marriage or cohabitation and years of schooling have low correlation with ISS-10 form ( $r_{s}$ from .13 to .27). Sex and marital status were independent based on ISS-10. 
Conclusion: The Index of Sexual Satisfaction, considered as key-factor to measure sexual satisfaction was reduced to 10 indicators, showing that the validity of evidence, is independent of being male or female, and it can be scaled using percentile scores rank.

Key words: sexual satisfaction, relationship satisfaction, marital aggrandizement, validity of tests, factor analysis, mexico.

\section{INTRODUCCIÓN}

Actualmente, la posibilidad de disfrutar de una actividad sexual acorde con las propias preferencias o deseos, libre de coacción, discriminación y violencia, se reconoce como un elemento nuclear de la salud sexual (Kismödi, Cottingham, Gruskin \& Miller, 2015). Este elemento subjetivo del disfrute es la base del concepto de satisfacción sexual, y esta última se puede definir como la respuesta que surge al evaluar los aspectos positivos y negativos asociados con las propias relaciones sexuales (Daugherty, Julian, Lynch, Chen, Whipple \& Ginsburg, 2016).

Ahora bien, en los estudios sobre la medición de la satisfacción sexual, se ha identificado un uso predominante de las escalas unidimensionales con múltiples ítems (Mark, Herbenick, Fortenberry, Sanders \& Reece, 2014). Entre estas, una de las más empleadas es el Índice de Satisfacción Sexual (ISS) de Hudson (1992), que fue desarrollado como una medición global del desacuerdo sexual y consta de 25 ítems. Fue creado a partir de la experiencia clínica de los autores, y refleja quejas comunes en los clientes sobre el componente sexual de su relación de pareja. Presenta, además, alta consistencia interna y validez discriminativa (Hudson, 1992), así como bajo sesgo en relación con la deseabilidad social (Muise, Stanton, Kim \& Impett, 2016).

EI ISS fue validado en México por Moral (2009a), quien, en una muestra incidental de 100 parejas, obtuvo una consistencia interna muy alta entre los 25 ítems del ISS ( $\alpha$ de Cronbach $=.91$ ), aunque el modelo de un factor mostró mal ajuste a los datos. Por esta razón fue simplificado a 10 indicadores (ítems $1,2,7,8,9,10,12,16,22$ y 25), conservándose entonces una consistencia interna muy alta, al tiempo que mejoró el ajuste y se logró que algunos índices alcanzaran valores aceptables. En otro estudio para esclarecer la estructura factorial del ISS, se trabajó con una muestra constituida por 807 participantes, seleccionados mediante muestreo de rutas al azar; en este caso se analizaron los datos a través de técnicas adecuadas para variables ordinales, como las correlaciones policóricas, el coeficiente alfa ordinal y el método de mínimos cuadrados libres de escala; de manera paralela, se calculó la validez convergente y discriminante de los factores. Los análisis validaron el modelo 
simplificado de un factor con 10 indicadores, aunque en lugar del ítem 7 se introdujo el ítem 3, al resultar mejor indicador. El ajuste a los datos fue bueno $\left(\chi^{2} / g I=1.108, \mathrm{GFI}=0.996, \mathrm{AGFI}=0.994, \mathrm{NFI}=0.995, \mathrm{RFI}=0.993, \mathrm{CFI}=0.999\right.$ y $S R M R=0.042$ ) y el factor único mostró consistencia interna muy alta ( $\alpha$ ordinal $=0.94$ ), así como validez convergente (coeficiente $\omega$ de McDonald = 0.97 y varianza media extraída $=0.65$ )

En este segundo estudio de validación del ISS (Moral, 2016), se comprobó la invarianza de los modelos factoriales, dividiendo de forma aleatoria la muestra en dos mitades, aunque no se contrastó la invarianza entre ambos sexos, a pesar de que existen diferencias en satisfacción sexual entre mujeres y hombres, y estas diferencias podrían ser relevantes (Castellanos, Álvarez, Ruiz \& Pérez, 2013). En una revisión sistemática, Sánchez, Santos y Sierra (2014) hallaron que una menor satisfacción se asocia con el hecho de ser mujer, pero esta asociación es más significativa y consistente en muestras clínicas que en muestras de población general o no clínicas; asimismo, señalan que la intimidad emocional tiene más impacto en la satisfacción sexual de las mujeres que en la de los hombres, al concebir la sexualidad como una práctica que rebasa lo biológico y se redefine en lo sociocultural (Ahumada, Lüttges, Molina \& Torres, 2014; Armenta-Hurtarte, SánchezAragón \& Díaz-Loving, 2014).

Por su parte, Veit, Stulhofer y Hald (2016) observaron que la falta de intimidad sexual es un determinante de mayor uso de medios de comunicación sexualmente explícitos en hombres, pero no en mujeres. $Y$ en un estudio longitudinal, Leavitt, McDaniel, Maas y Feinberg (2016) encontraron que el estrés parental influye en la satisfacción sexual de la madre, pero no en la del padre. Los autores atribuyen estos resultados a los roles socialmente construidos para ambos géneros en la sociedad y, particularmente, en la familia, así como a alteraciones en la relación de pareja ante el estrés sufrido por la madre. En una línea de investigación llevada a cabo con estudiantes universitarios colombianos, Díaz, Bulla y Moreno (2016) encontraron que el exceso de tareas y la falta de tiempo generaban más quejas de fatiga, falta de deseo y satisfacción sexual en las mujeres que en los hombres, por lo que el estrés académico afectaba más a las mujeres que a los hombres. Por otra parte, en estudiantes universitarios argentinos, hallaron que la asociación de la percepción de intimidad con la satisfacción sexual y con la relación era mayor en mujeres que en hombres. 
Aunque el sexo es un correlato significativo de la satisfacción marital, mayores efectos aún tienen la edad y la duración de la relación; de modo que la satisfacción sexual disminuye al relacionarse con una mayor edad y más años de matrimonio (Fallis, Rehman, Woody \& Purdon, 2016; Sánchez et al., 2014). También el mayor número de hijos tiene un efecto decrementador (McNulty, Wenner \& Fisher, 2016). En contraposición con lo anterior, la asociación de una mayor escolaridad con una mayor satisfacción sexual varía en su significación de un estudio a otro (Kettrey \& Carpenter, 2015), mientras que el hecho de que las parejas estén casadas o vivan en unión libre no parece tener efecto significativo (Sánchez et al., 2014).

Diversos estudios han hallado relación entre la satisfacción sexual y la satisfacción marital, con asociaciones altas tras eliminar los ítems de contenido sexual en las escalas de satisfacción marital. Moral (2011) halló correlaciones moderadas entre la satisfacción sexual y el engrandecimiento marital tanto en hombres como en mujeres, y observó que un componente de enamoramiento (definido por ajuste diádico, satisfacción con la relación y engrandecimiento marital) predecía la satisfacción sexual con un tamaño de efecto mediano; asimismo, Liu, Wang y Jackson (2017) y Zhou, Chiu y Chui (2017) corroboraron en parejas chinas la importancia del engrandecimiento marital en la satisfacción con la relación de pareja, incluida la vida sexual.

Debido a la utilidad que proporciona en los estudios de salud sexual y pareja contar con una medición breve y válida de la satisfacción sexual (Ahumada et al., 2014; Díaz et al., 2016), el propósito del presente trabajo consistió en aportar nuevas pruebas de validez del ISS como una medición unidimensional de la satisfacción sexual reducida a 10 indicadores (ISS-10) y describir su distribución en parejas. Como objetivos se plantearon: contrastar la invarianza del modelo de un factor entre ambos sexos; describir la distribución del ISS-10; aportar pruebas de validez convergente en relación con la satisfacción con la relación y el engrandecimiento marital; y comprobar el efecto del sexo, la edad, el tiempo de casados o unión libre, el número de hijos, la escolaridad y el estado civil sobre las puntuaciones en el ISS-10.

Se esperaba que el modelo de un factor mostrase buenas propiedades de invarianza entre ambos sexos; una distribución con asimetría hacia el polo de la satisfacción, como aparece en las escalas que evalúan aspectos de satisfacción con la relación de pareja o familiar (Cheng \& Smyth, 2015; Moral, 2009b); una asociación positiva y alta con la satisfacción con la relación (McNulty et al., 2016; Sánchez et al., 2014) y en menor cuantía con el engran- 
decimiento marital (Liu et al., 2017; Moral, 2011; Zhou et al., 2017); un tamaño de efecto mediano o pequeño sobre el ISS-10 de la edad, el tiempo de casados o en unión libre y el número de hijos, pero pequeño o trivial del sexo, el estado civil y la escolaridad (Fallis et al., 2016; Kettrey \& Carpenter, 2015; Sánchez at al., 2014).

\section{MÉTODO}

\subsection{Diseño}

El presente estudio es una investigación instrumental. En esta categoría se incluyen los estudios enfocados en el desarrollo de pruebas, incluyendo su diseño y adaptación (Ato, López \& Benavente, 2013). Para estudiar aspectos de consistencia interna, invarianza factorial entre sexos y distribución de la escala, se usó un diseño ex post facto transversal, al no requerirse manipulación de variables ni seguimiento de casos (Breakwell, Smith \& Wright, 2012).

\subsection{Participantes}

Los criterios de inclusión en la investigación fueron: tener al menos 18 años, estar casado o vivir en unión libre con una pareja del sexo opuesto, residir en la ciudad de Monterrey (México), saber leer y escribir, y prestar el consentimiento informado. Los criterios de eliminación fueron: tramitación incompleta del cuestionario e informar que la pareja estuvo presente en el momento de ser contestado, leyendo o preguntando por las respuestas.

Se empleó el muestreo de rutas al azar, recolectándose en total una muestra de 807 participantes con todos sus datos completos. De ellos, 431 (53.4 \%) fueron mujeres y 376 (46.6\%) hombres, sin diferencia de frecuencia estadísticamente significativa entre ambos sexos por la prueba binomial $(p=0.057)$. Con respecto al estado civil, 727 (90\%) de los 807 participantes indicaron estar casados y 80 (10 \%) en unión libre. La media de años de convivencia fue de 11.61 ( $D E=9.33)$, variando de 6 meses a 43 años. Por otra parte, de las 807 personas encuestadas, 677 (84\%) dijeron tener hijos y 130 (16\%), no. La media de hijos fue de $1.99(D E=1.30)$, variando de 0 a 9. La media de edad fue 35.40 años $(D E=10.41)$ y la mediana 34 , variando de 18 a 94 años. La mediana de escolaridad $(M d n=2)$ correspondió a secundaria (de siete a nueve años de escolaridad) y la media $(M=2.51)$ quedó entre estudios de secundaria y media superior (en torno a 10 años de escolaridad). La mediana y la media de ingreso mensual de la familia correspondieron al rango de 6000 a 11.999 pesos. Con respecto a la religión, 662 de los 807 participantes (82 \%) 
dijeron ser cristianos católicos, 76 (9.4 \%) cristianos no católicos, 13 (1.6 \%) pertenecer a otras religiones y 56 (6.9\%). a ninguna.

En la población total de Monterrey se reporta 98 hombres por cada 100 mujeres, una mediana de 34 al eliminar al $31 \%$ de la población menor de 18 años, una media de 9.5 años de escolaridad (entre primer semestre y segundo de educación media superior), una media de ingresos mensuales de 6.700 pesos mexicanos, una media de 2 hijos, y el $83 \%$ de la población con afiliación religiosa cristiana católica, $7.4 \%$ cristiana no católica, $2.5 \%$ de otra religión y 6.9 \% sin religión (Instituto Nacional de Estadística, Geografía e Informática, 2015). Estos datos poblacionales son estadísticamente equivalentes a los de la muestra en la frecuencia de ambos sexos $\left(\chi^{2}[1, N=807]=\right.$ $1.87, p=0.171)$, de la afiliación religiosa $\left(\chi^{2}[3, N=807]=7.13, p=0.068\right)$ y en la media de hijos $(t[806]=-0.27, p=0.787)$, o muy semejantes, como en la mediana de edad, de escolaridad y de ingresos. Si se reduce la población de la zona metropolitana de Nuevo León a la de adultos casados y en unión libre, se tendría $89 \%$ casados y $11 \%$ en unión libre, esto es, nueve personas casadas por cada persona en unión libre, lo que es un porcentaje equivalente al muestral $\left(\chi^{2}[1, N=807]=0.97, p=0.324\right)$.

\subsection{Instrumentos}

Como ya se ha dicho, el Índice de Satisfacción Sexual (ISS; Hudson, 1992) fue adaptado a la población mexicana por Moral (2009a). Está integrado por 25 ítems tipo Likert con un recorrido de uno a siete, y por tres categorías ordenadas en cada polaridad (satisfacción-insatisfacción) y una intermedia: 1 = en ningún momento, 2 = en muy raras ocasiones, 3 = algunas veces, 4 = parte del tiempo, 5 = buena parte del tiempo, 6 = la mayor parte del tiempo, y $7=$ en todo momento. Doce de los 25 ítems están redactados en sentido de la satisfacción sexual $(1,2,3,9,10,12,16,17,19,21,22$ y 23$)$ y los otros 13 en sentido de la insatisfacción sexual. Conforme a los resultados del estudio de Moral (2016), se eliminaron quince ítems (cuatro de satisfacción y once de insatisfacción), resultando el ISS-10. Las puntuaciones en el ISS-10 se obtienen al sumar los ítems $1,2,3,8,9,10,12,16,22$ y 25 , tras haber invertido las puntuaciones en los ítems 8 y 25 ( 8 - valor de respuesta al ítem), y dividiendo por el número de ítems sumados: ISS-10 = (ítem $1+$ ítem $2+$ ítem $3+$ [ 8 - ítem8 8 + ítem $9+$ ítem10 + ítem 12 + ítem 16 + ítem $22+$ [ 8 - ítem25]) / 10. El rango del ISS-10 varía en un intervalo continuo de 1 a 7 , de modo que una mayor puntuación refleja más satisfacción sexual. La consistencia interna de los 10 ítems fue muy alta con un coeficiente $\alpha$ ordinal de .94 en la presente muestra (Moral, 2016). 
La Escala de Valoración de la Relación (RAS) (Hendrick, 1988) fue adaptada a población mexicana por Moral (2015). Está integrada por siete ítems tipo Likert con un rango ordinal de respuestas de uno a cinco. Las puntuaciones en la RAS se obtienen por suma simple de los ítems. Dos ítems están redactados en sentido contrario a la satisfacción con la relación (ítems 4 y 7), por lo que, antes de ser sumados, hay que invertir sus puntuaciones (6 - valor de respuesta al ítem). El rango de la escala varía de 7 a 35 . Una mayor puntuación refleja una mayor satisfacción con la relación de pareja. En la muestra mexicana, que corresponde a la del presente estudio, la consistencia interna de la RAS por sexo fue: en mujeres ( $\alpha$ ordinal $=0.94$ ) y hombres ( $\alpha$ ordinal $=0.92$ ). El análisis paralelo de Horn y la prueba de la correlación parcial media mínima de Velicer sugirieron una solución de un factor. El modelo de un factor (con una correlación entre los residuos de los dos ítems inversos) tuvo buen ajuste a los datos y sus propiedades de invarianza por sexo fueron aceptables en cuanto al método de mínimos cuadrados no ponderados (Moral, 2015).

Por último, la Escala de Engrandecimiento Marital (MAS) (O'Rourke \& Cappeliez, 2002) fue adaptada a la población mexicana por Moral (2009b). Evalúa el posible sesgo de exagerar las cualidades positivas y minimizar las negativas al momento de reportar la satisfacción con la relación y la pareja. Consta de 18 ítems tipo Likert en un rango de respuestas de 1 totalmente en desacuerdo a 7 totalmente de acuerdo. Catorce de sus 18 ítems están redactados en atención al rasgo del engrandecimiento, y cuatro en sentido opuesto (ítems 2, 11, 13 y 17). Conforme a los resultados del estudio de Moral (2016), se eliminaron cuatro ítems (el ítem directo 7 y los ítems inversos 11, 13 y 17), con lo que resultó la MAS-14. Las puntuaciones en la MAS-14 se obtienen al sumar el valor numérico que corresponde a la respuesta dada a cada ítem, tras invertir este valor en el ítem 2 ( 8 - valor de respuesta), y dividiendo por el número de ítems sumados (14), lo que proporciona puntuaciones en un rango continuo de 1 a 7 . En la presente muestra, la MAS-14 tuvo una consistencia interna alta ( $\alpha$ ordinal $=0.87$ ); y el modelo de un factor con 14 indicadores y todos sus residuos de medida independientes tuvo buen ajuste a los datos y adecuadas propiedades de invarianza entre ambos sexos. La distribución de la MAS-14 mostró sin embargo una ligera asimetría negativa, no ajustándose a una curva normal.

\subsection{Procedimiento}

De forma aleatoria se seleccionaron 81 direcciones a partir de la Guía Roji (dos direcciones por encuestador) y cada dirección se tomó como punto 
de partida de un itinerario. A su vez, en cada itinerario se recolectaron 10 unidades (prestado el consentimiento informado y con datos completos). Se buscó que fueran cinco mujeres y cinco hombres independientes (uno por casa) en cada itinerario. El encuestador tocaba el timbre o puerta de la casa. Si esta era abierta, se presentaba como un ayudante de investigación, se informaba de los objetivos del estudio (estudio sobre dinámica de la vida marital o de convivencia por medio de un cuestionario), del responsable del mismo y criterios de inclusión. Si la persona deseaba participar voluntariamente y satisfacía los criterios de inclusión, se dejaba un cuestionario para recogerlo media hora después o cuando lo indicase la persona. Se instruía que debía contestarlo a solas, sin que su pareja leyese las preguntas o respuestas. Al recogerlo, se preguntaba si la pareja había estado presente a la hora de contestar y sí había visto sus respuestas; en caso de que así hubiese sucedido las respuestas respectivas no se incluían en la base de datos. En caso de que no, se revisaba que estuviera contestado; si había preguntas sin respuesta, se pedía amablemente que completara el instrumento.

Los entrevistadores fueron alumnos de primer semestre de la Facultad de Psicología de la Universidad Autónoma de Nuevo León (UANL) que cursaban la materia de Investigación en Psicología. Recibieron entrenamiento por parte del responsable del proyecto de investigación para colectar los casos y fueron compensados con puntos extras en su calificación.

Para determinar el tamaño de la muestra se usó la fórmula de población de tamaño infinito o desconocido, al ser el tamaño poblacional mayor que 100.000 (García, Reding \& López, 2013). Así, bajo un modelo de curva normal, si se desea estimar la media del ISS con una desviación estándar esperada de 14.64 en un rango de 0 a 100 (Moral, 2009a) y con un error absoluto de estimación de 1.011, se requeriría una muestra mínima de 806 individuos.

La proporción de respuesta fue aproximadamente de 7 por cada 10 casas contactadas. Se eliminaron 44 cuestionarios por datos incompletos. Junto a los tres instrumentos usados en el presente artículo, se administraron cuatro escalas que evalúan actitud hacia la infidelidad, infidelidad, afecto positivo y negativo y depresión, cuyos datos serán analizados en posteriores trabajos. Todos los cuestionarios aplicados terminaban con la escala de depresión, a fin de evitar el posible sesgo hacia una autoevaluación negativa que pudiera inducir. Permutando los seis instrumentos restantes se definieron 720 secuencias. Se aplicaron cada una de estas 720 secuencias, y se escogieron 
al azar 131 de ellas para completar la muestra (807 completos y 44 incompletos). La muestra fue reclutada de enero a mayo de 2014.

El estudio fue aprobado en sus aspectos éticos por el Comité de Doctorado, autoridad pertinente dentro de la Facultad de Psicología de la UANL, a pesar de no formar parte de ninguna tesis doctoral, debido a que fue sometido de forma extraordinaria por el responsable del estudio para cumplir requisitos del programa de financiamiento, que estuvo en cabeza del Programa de Apoyo a la Investigación Científica y Tecnológica de la UANL. En la primera hoja del cuestionario, se solicitó el consentimiento informado de los participantes, garantizándose el anonimato de las respuestas y se informó sobre la identidad del responsable del estudio, con quien se podía contactar por correo electrónico para cualquier cuestión suscitada por el estudio. No se solicitó ningún dato de identificación personal. De este modo, se respetaron las normas éticas de investigación de la Secretaría de Salud (2013), Sociedad Mexicana de Psicología (2007) y Asociación Americana de Psicología (2016).

\subsection{Análisis de datos}

Para comprobar la invarianza del modelo de un factor entre ambos sexos, se usó análisis factorial confirmatorio multigrupo. Se definieron cuatro modelos anidados en restricciones. La función de discrepancia se estimó por mínimos cuadrados libres de escala (SLS), y el ajuste se valoró usando la chi-cuadrada relativa $\left(\chi^{2} / g l\right)$, la prueba de muestreo repetitivo de Bollen-Stine con la extracción de 500 muestras $\left(p_{B S}\right)$, el índice de bondad de ajuste sin ajustar $(G F I)$ y ajustado $(A G F I)$, el índice normado de ajuste $(N F I)$, el índice relativo de ajuste o coeficiente $\rho_{1}$ de Bollen (RFI), el índice comparativo de ajuste $(C F I)$ y el residuo estandarizado cuadrático medio (SRMR). Siguiendo a Byrne (2016), se consideraron valores de buen ajuste: $\chi 2 / \mathrm{gl} \leq 2, \mathrm{pBS}>0.05, \mathrm{GFI}, \mathrm{NFI}, \mathrm{RFI}$ y $\mathrm{CFI} \geq 0.95, \mathrm{AGFI} \geq 0.90$ y SRMR $\leq 0.05$; $y$ adecuados: $\chi 2 / \mathrm{gl} \leq 3, \mathrm{pBS}>0.01, \mathrm{GFI}$, $\mathrm{NFI}, \mathrm{RFI}$ y $\mathrm{CFI} \geq 0.90, \mathrm{AGFI} \geq 0.85$ y $\mathrm{SRMR}<0.10$. Se interpretó que la bondad de ajuste de un modelo es mayor que la del otro cuando $|\Delta \chi 2| /|\Delta \mathrm{gl}|>3$ y $\Delta \mathrm{GFI}$, $\Delta \mathrm{NFI}, \Delta \mathrm{CFI}$ y $\Delta \mathrm{RFI}>0.01$ (Byrne, 2016). La equivalencia de parámetros entre las dos muestras se determinó por el solapamiento de los intervalos con una confianza de $95 \%$, en tanto que los intervalos se calcularon por el método de percentiles corregidos de sesgo con la extracción de 500 muestras, y por este mismo método se contrastó la significación de los parámetros.

El ajuste a una distribución normal se contrastó por la prueba de Kolmogorov-Sminrnov-Lilliefors. Y debido a la falta de normalidad, la comparación de medias por sexo y estado civil se realizó mediante la prueba $U$ de 
Mann-Whitney y el tamaño del efecto se estimó por medio del coeficiente de correlación, usando la siguiente fórmula: $r=Z_{u} /(N)^{1 / 2}$ (Kerby, 2014). La relación del ISS-10 con la RAS, y la MAS-14, edad, años de casados o en unión libre, número de hijos y escolaridad se estimó por el coeficiente de correlación por rangos de Spearman (rS). La sustantividad de la relación del ISS-10 se comprobó con la RAS, parcializando la MAS-14; con la MAS, parcializando la RAS; y con las seis variables demográficas, parcializando la MAS-14 y la RAS. Para este objetivo se usó la fórmula de parcialización de Fisher, introduciendo coeficientes de correlación de rangos de Spearman: $r p=r S 12 \bullet 3=$ (rS 12 - rS 13 * rS 23) / ([1- rS 132]1/2 * [1- rS 132]1/2)) (Corder \& Foreman, 2014). A partir de ello, los valores de $r S$ y rp menores de 0.10 se interpretaron como un tamaño de efecto o fuerza de asociación trivial, así: de 0.10 a 0.299 pequeño, de 0.30 a 0.499 mediano, de 0.50 a 0.699 grande y mayores que 0.70 , muy grande (Corder \& Foreman, 2014). Se comprobaron efectos de interacción del sexo con las otras variables demográficas sobre el ISS-10 por la prueba de rangos alineados (Oliver, González \& Rosel, 2014). Los cálculos se realizaron con SPSS versión 22, el menú $\mathrm{R}$ versión 2.4 para SPSS22, y los programas de Excel 2007 y AMOS versión 16.

\subsection{Conflictos de interés}

El autor declara que en desarrollo de este trabajo no se presentó ningún conflicto de intereses.

\section{RESULTADOS}

\subsection{Invarianza entre ambos sexos del modelo de un factor con 10 indicadores}

En general, los índices del modelo sin restricciones mostraron buen ajuste, salvo la prueba de Bollen-Stine que reflejó un ajuste aceptable $\left(p_{B S}=10 / 499\right.$ $=0.020$ ). Igual ocurrió en el modelo con restricciones en los pesos de medida $\left(p_{B S}=11 / 499=0.022\right)$. La equivalencia de la bondad de ajuste entre estos dos modelos fue buena por $\Delta \mathrm{GFI}, \Delta \mathrm{NFI}, \Delta \mathrm{CFI}$ y $\Delta \mathrm{RFI}$, y aceptable por $|\Delta \mathrm{x} 2| /|\Delta \mathrm{g}| \mid$. En el modelo con restricciones en la varianza estructural, la bondad de ajuste fue buena por seis de los ocho y aceptable por $\chi^{2} / g l$ y $S R M R$. Y el modelo con restricciones en las varianzas de los residuos, la bondad de ajuste fue buena por todos los índices, salvo aceptable por $\chi^{2} / g l$. La equivalencia de la bondad de ajuste entre estos dos últimos modelos fue buena como evidencian los cinco índices diferenciales (Tabla 1). En todos estos modelos, los residuos de medida se especificaron de forma independiente. 
Tabla 1

Índices de bondad de ajuste en el contraste multigrupo (mujeres y hombres)

\begin{tabular}{|c|c|c|c|c|c|c|c|}
\hline \multirow{2}{*}{$\begin{array}{l}\text { MODELOS } \\
\text { ANIDADOS }\end{array}$} & \multirow{2}{*}{$\begin{array}{l}\text { ÍNDICES } \\
\text { DE AJUSTE }\end{array}$} & \multicolumn{2}{|c|}{ INTERPRETACIÓN } & \multicolumn{4}{|c|}{ MODELOS ANIDADOS } \\
\hline & & Bueno & Aceptable & $S R$ & RPM & RVE & RRM \\
\hline & $x^{2}$ & & & 102.739 & 128.841 & 183.956 & 192.531 \\
\hline & $\mathrm{g} \mid$ & & & 70 & 79 & 80 & 90 \\
\hline & $x 2 / g \mid$ & $\leq 2$ & $\leq 3$ & 1.468 & 1.631 & 2.299 & 2.139 \\
\hline & $\mathrm{pBS}$ & $>0.05$ & $>0.01$ & 0.020 & 0.022 & 0.062 & 0.072 \\
\hline & GFI & $\geq 0.95$ & $\geq 0.90$ & 0.994 & 0.993 & 0.990 & 0.989 \\
\hline & AGFI & $\geq 0.90$ & $\geq 0.85$ & 0.991 & 0.990 & 0.986 & 0.987 \\
\hline & $\mathrm{NFI}$ & $\geq 0.95$ & $\geq 0.90$ & 0.993 & 0.991 & 0.987 & 0.986 \\
\hline & $\mathrm{CFI}$ & $\geq 0.95$ & $\geq 090$ & 0.998 & 0.996 & 0.992 & 0.993 \\
\hline & RFI & $\geq 0.95$ & $\geq 0.90$ & 0.990 & 0.989 & 0.985 & 0.986 \\
\hline & SRMR & $\leq 0.05$ & $\leq 0.09$ & 0.039 & 0.045 & 0.057 & 0.050 \\
\hline & $|\Delta \times 2| /|\Delta g| \mid$ & $\leq 2$ & $\leq 3$ & & 2.900 & 8.122 & 4.490 \\
\hline & $\Delta \mathrm{GFI}$ & $<0.01$ & & & 0.001 & 0.004 & 0.005 \\
\hline \multirow[t]{5}{*}{ SR } & $\triangle N F I$ & $<0.01$ & & & 0.002 & 0.006 & 0.006 \\
\hline & $\Delta \mathrm{CFI}$ & $<0.01$ & & & 0.001 & 0.005 & 0.005 \\
\hline & $\triangle \mathrm{RFI}$ & $<0.01$ & & & 0.001 & 0.005 & 0.004 \\
\hline & $|\Delta \times 2| /|\Delta g| \mid$ & $\leq 2$ & $\leq 3$ & & & 55.115 & 5.790 \\
\hline & $\Delta \mathrm{GFI}$ & $<0.01$ & & & & 0.003 & 0.004 \\
\hline \multirow[t]{5}{*}{ RPM } & $\Delta N F I$ & $<0.01$ & & & & 0.004 & 0.005 \\
\hline & $\Delta \mathrm{CFI}$ & $<0.01$ & & & & 0.004 & 0.004 \\
\hline & $\triangle \mathrm{RFI}$ & $<0.01$ & & & & 0.004 & 0.003 \\
\hline & $|\Delta \times 2| /|\Delta \mathrm{g}| \mid$ & $\leq 2$ & $\leq 3$ & & & & 0.858 \\
\hline & $\Delta \mathrm{GFI}$ & $<0.01$ & & & & & 0.001 \\
\hline \multirow[t]{3}{*}{ RVC } & $\Delta N F I$ & $<0.01$ & & & & & 0.001 \\
\hline & $\Delta \mathrm{CFI}$ & $<0.01$ & & & & & -0.001 \\
\hline & $\Delta \mathrm{RFI}$ & $<0.01$ & & & & & -0.001 \\
\hline
\end{tabular}

Muestras: 431 mujeres y 376 hombres. Método: Mínimos Cuadrados Libres de Escala. Modelos anidados: $\mathrm{SR}=$ sin restricciones, $\mathrm{RPM}=$ con restricciones en los pesos de medida, $\mathrm{RVE}=$ con restricción en la varianza estructural y RRM = con restricciones en las varianzas de los residuos de medida.

Todos los parámetros fueron significativos y equivalentes entre mujeres y hombres con una $p>0.05$, como refleja el solapamiento de los intervalos estimados. El tamaño de efecto del factor sobre sus 10 indicadores $(\lambda)$ varió de grande (ítem 8) a muy grande (restantes ítems), con una varianza media extraída mayor que 0.50 y una fiabilidad compuesta (por el coeficiente omega de McDonald) mayor que 0.80 en todos los modelos y en las dos muestras. En la Tabla 2 pueden apreciarse los parámetros del modelo sin restricciones en mujeres y hombres. 
Tabla 2

Parámetros del modelo sin constricciones

\begin{tabular}{|c|c|c|c|c|}
\hline \multirow{2}{*}{ VAR. } & MUJER & HOMBRE & MUJER & HOMBRE \\
\hline & $\Lambda$ [IC DEL $95 \%]$ & $\Lambda$ [IC DEL $95 \%]$ & S2D [IC DEL $95 \%]$ & S2 2 [IC DEL $95 \%]$ \\
\hline 1 & $0.815^{* *}[0.747,0.867]$ & $0.813^{* *}[0.764,0.853]$ & $0.893^{* *}[0.655,1.163]$ & $0.859 * *[0.710,1.051]$ \\
\hline 2 & $0.887^{* *}[0.851,0.913]$ & $0.852^{* *}[0.808,0.891]$ & $0.624^{* *}[0.486,0.779]$ & $0.726^{* *}[0.560,0.942]$ \\
\hline 3 & $0.856^{* *}[0.821,0.888]$ & $0.868^{* *}[0.831,0.899]$ & $0.942^{* *}[0.755,1.168]$ & $0.753^{* *}[0.593,0.939]$ \\
\hline 8 & $-0.603 * *[-0.693,-0.493]$ & $-0.553 * *[-0.657,-0.446]$ & $1.676^{* *}[1.289,2.205]$ & $1.705^{* *}[1.307,2.271]$ \\
\hline 9 & $0.765^{* *}[0.700,0.817]$ & $0.779 * *[0.723,0.828]$ & $1.435^{* *}[1.142,1.791]$ & $1.301^{* *}[1.017,1.691]$ \\
\hline 10 & $0.847^{* *}[0.803,0.884]$ & $0.795 * *[0.741,0.841]$ & $0.954^{* *}[0.735,1.187]$ & $1.152^{* *}[0.901,1.425]$ \\
\hline 12 & $0.856^{* *}[0.800,0.899]$ & $0.861^{* *}[0.806,0.899]$ & $0.986^{* *}[0.717,1.394]$ & $0.864 * *[0.653,1.234]$ \\
\hline 16 & $0.846^{* *}[0.799,0.880]$ & $0.773 * *[0.704,0.827]$ & $1.075^{* *}[0.858,1.371]$ & $1.400 * *[1.076,1.837]$ \\
\hline 22 & $0.736 *[0.647,0.789]$ & $0.820^{* *}[0.769,0.857]$ & $1.303^{* *}[1.033,1.717]$ & $0.871^{* *}[0.708,1.117]$ \\
\hline \multirow[t]{2}{*}{25} & $-0.704 * *[-0.767,-0.611]$ & $-0.546^{* *}[-0.661,-0.427]$ & $1.220 * *[0.937,1.644]$ & $1.937^{* *}[1.460,2.616]$ \\
\hline & & & S2F [IC del $95 \%]$ & S2F [IC del $95 \%]$ \\
\hline $\mathrm{F}$ & & & $1.761^{* *}[1.411,2.139]$ & $1.668^{* *}[1.328,2.009]$ \\
\hline
\end{tabular}

(**) Muestras: 431 mujeres y 376 hombres. Método para estimar los parámetros: Mínimos Cuadrados Libres de Escala. Método para calcular los intervalos de confianza: percentiles corregidos de sesgo. Var. $=$ variables,$\lambda=$ peso estructural, $\mathrm{S} 2 \delta$ = varianza del residuo, $\mathrm{S} 2=$ varianza del factor, $\mathrm{IC}=$ intervalo de confianza y $\mathrm{F}$ = factor único.

\subsection{DESCRIPCIÓN DE LA DISTRIBUCIÓN}

La media del ISS-10 fue de 5.33 (IC del 95: 5.23, 5.43) con un rango de 1 a 7 y su desviación estándar de 14.04. La distribución de las puntuaciones en el ISS-10 presentó asimetría negativa $(A=-0.88$, IC del $95:-1.05,-0.71)$, y no se ajustó a una curva normal $(\max |D|=0.14, p<0.001)$. Sus deciles y cuartiles se pueden ver en la Tabla 3.

Tabla 3

Deciles y cuartiles del ISS-10 (con un rango de 1 a 7 )

\begin{tabular}{cccccccc}
\hline \multirow{2}{*}{ ESTADÍSTICOS } & MUESTRAL & \multicolumn{3}{c}{ GRUPOS DE EDAD } & \multicolumn{3}{c}{ AÑOS DE CASADOS O UNIÓN LIBRE } \\
\cline { 3 - 8 } & TOTAL & $\mathbf{1 8 - 2 9}$ & $\mathbf{3 0 - 3 9}$ & $\mathbf{4 0 - 4 9}$ & $\mathbf{2 5 0}$ & $\mathbf{9 9}$ & $\mathbf{9}$ \\
\hline N & 807 & 285 & 230 & 220 & 72 & 402 & 405 \\
D1 = P10 & 3.3 & 3.9 & 3.4 & 2.9 & 2.4 & 3.6 & 2.9 \\
D2 = P20 & 4.1 & 4.9 & 4.0 & 3.7 & 3.5 & 4.6 & 3.8 \\
Q1 = P25 & 4.4 & 5.1 & 4.4 & 3.9 & 3.8 & 4.975 & 4.0 \\
D3 = P30 & 4.7 & 5.3 & 4.63 & 4.2 & 4.1 & 5.2 & 4.3 \\
D4 = P40 & 5.2 & 5.9 & 5.3 & 4.6 & 4.6 & 5.7 & 4.8 \\
Q2 = D5 = P50 & 5.8 & 6.2 & 5.8 & 5.1 & 5.2 & 6.1 & 5.3 \\
D6 = P60 & 6.1 & 6.4 & 6.1 & 5.5 & 5.7 & 6.3 & 5.8 \\
D7 = P70 & 6.3 & 6.6 & 6.4 & 6.0 & 5.9 & 6.6 & 6.1 \\
Q2 = P75 & 6.4 & 6.7 & 6.5 & 6.2 & 6.0 & 6.6 & 6.2 \\
D8 = P80 & 6.6 & 6.7 & 6.6 & 6.3 & 6.2 & 6.7 & 6.3 \\
D9 = P90 & 6.9 & 7.0 & 6.9 & 6.6 & 6.4 & 6.9 & 6.7 \\
\hline
\end{tabular}

$\mathrm{N}=$ tamaño de la muestra, $\mathrm{D}=$ Deciles, $\mathrm{Q}=$ cuartiles y $\mathrm{P}=$ Percentiles. 


\subsection{Validez convergente}

En la muestra total y de mujeres, la correlación entre el ISS-10 y la RAS fue muy alta, y en la de hombres, alta. En las tres muestras, la correlación entre el ISS-10 y la MAS-14 fue también alta. Todas ellas fueron significativas (Tabla 3). Así, al calcular los errores estándar de los coeficientes de correlación con la fórmula: $\left.E E=\left(\left[1-r_{s}^{2}\right] /[n-2]\right)^{1 / 2}\right)$ y definir intervalos de confianza del $95 \%$ $\left(r_{s} \pm t_{n-2} * E E\right)$, se observó un claro solapamiento de los valores entre mujeres y hombres, indicando equivalencia estadística con una $p>0.05$ (Tabla 4).

Debido a que el engrandecimiento marital constituye una potencial fuente de sesgo, su efecto sobre la satisfacción con la relación se controló. No obstante, tras parcializar la varianza de la MAS-14, la correlación entre el ISS-10 y la satisfacción con la relación (RAS) siguió siendo significativa, alta y positiva en las tres muestras, con una varianza compartida mayor que $27 \%$ y menor que $35 \%$ (Tabla 4).

A su vez, al parcializar la varianza de la satisfacción con la relación (RAS), en la correlación entre el ISS-10 y la MAS-14, esta correlación siguió siendo significativa y positiva, aunque disminuyó a baja en las tres muestras, con una varianza compartida mayor que $3 \%$ y menor que $5 \%$ (Tabla 4 ).

Tabla 4

Correlaciones por el coeficiente de rangos de Spearman

\begin{tabular}{|c|c|c|c|}
\hline \multirow{3}{*}{$\begin{array}{l}\text { ESCALAS Y VARIABLES } \\
\text { DEMOGRÁFICAS }\end{array}$} & \multicolumn{3}{|c|}{ ISS-10 } \\
\hline & TOTAL (N = 807) & MUJERES ( $N=431)$ & HOMBRES ( $N=376)$ \\
\hline & rS [IC del $95 \%$ ] & rS [IC del $95 \%$ ] & rS [IC del $95 \%$ ] \\
\hline RAS & $0.713^{* * *}[0.664,0.762]$ & $0.732 * * *[0.667,0.797]$ & $0.690^{* * *}[0.616,0.764]$ \\
\hline MAS-14 & $0.559 * * *[0.502,0.616]$ & $0.565 * * *[0.487,0.643]$ & $0.556^{* * *}[0.471,0.641]$ \\
\hline Edad & $-0.262 * * *[-0.329,-0.195]$ & $-0.296 * * *[-0.387,-0.205]$ & $-0.220 * * *[-0.319,-0.121]$ \\
\hline Tiempo de casados & $-0.267 * * *[-0.334,-0.200]$ & $-0.271^{* * *}[-0.362,-0.180]$ & $-0.266 * * *[-0.364,-0.168]$ \\
\hline Número de hijos & $-0.246^{* * *}[-0.313,-0.179]$ & $-0.263 * * *[-0.355,-0.171]$ & $-0.225 * * *[-0.324,-.0126]$ \\
\hline Escolaridad & $0.130 * * *[0.061,0.199]$ & $0.135 * *[0.041,0.229]$ & $0.129 *[0.028,0.230]$ \\
\hline \multicolumn{4}{|c|}{ Parcializando la MAS-14 } \\
\hline RAS & $0.560 * * *[0.503,0.617]$ & $0.586 * * *[0.509,0.663]$ & $0.526 * * *[0.440,0.612]$ \\
\hline Edad & $-0.269 * * *[-0.336,-0.202]$ & $-0.291 * * *[-0.382,-0.200]$ & $-0.236 * * *[-0.335,-0.137]$ \\
\hline Tiempo de casados & $-0.250 * * *[-0.317,-0.183]$ & $-0.240 * * *[-0.332,-0.148]$ & $-0.266 * * *[-0.364,-0.168]$ \\
\hline Número de hijos & $-0.195 * * *[-0.263,-0.127]$ & $-0.208^{* * *}[-0.301,-0.115]$ & $-0.174 * * *[-0.274,-0.074]$ \\
\hline Escolaridad & $0.080 *[0.011,0.149]$ & $0.088 n s[-0.007,0.183]$ & $0.082 \mathrm{~ns}[-0.019,0.183]$ \\
\hline \multicolumn{4}{|c|}{ Parcializando la RAS } \\
\hline MAS-14 & $0.197 * * *[0.129,0.265]$ & $0.189 * * *[0.096,0.282]$ & $0.215^{* * *}[0.116,0.314]$ \\
\hline Edad & $-0.321 * * *[-0.387,-0.255]$ & $-0.326 * * *[-0.416,-0.236]$ & $-0.319 * * *[-0.415,-0.223]$ \\
\hline Tiempo de casados & $-0.305^{* * *}[-0.371,-0.239]$ & $-0.305 * * *[-0.395,-0.215]$ & $-0.318 * * *[-0.414,-0.222]$ \\
\hline Número de hijos & $-0.196 * * *[-0.264,-0.128]$ & $-0.227 * * *[-0.319,-0.135]$ & $-0.167 * *[-0.267,-0.067]$ \\
\hline Escolaridad & $0.091^{* *}[0.022,0.160]$ & 0.090 ns $[-0.005,0.185]$ & $0.099 n s[-0.002,0.200]$ \\
\hline
\end{tabular}

$* * * \mathrm{p}<0.001,{ }^{* *} \mathrm{p}<0.01$. Intervalo de confianza del $95 \%$. 


\subsection{Efecto de las variables sociodemográficas sobre el ISS-10}

La tendencia central de la distribución del ISS-10 fue estadísticamente equivalente $\left(Z_{u}=-0.93, p=0.352 ; r=-0.03\right)$ entre mujeres $(M=5.35$ [IC del 95: 5.21, 5.49]: $\left.M d n=5.8\left[Q_{1}=4.5, Q_{2}=6.5\right]\right)$ y hombres ( $M=5.31$ [IC del 95: 5.17, 5.45]: $\left.M d n=5.8\left[Q_{1}=4.4, Q_{2}=6.4\right]\right)$. Por tanto, el sexo y la satisfacción sexual fueron dos variables independientes.

El estado civil (casados y en unión libre) tampoco tuvo efecto sobre la satisfacción sexual (ISS-10) en la muestra total $\left(Z_{u}=-0.225, p=0.822 ; r=-0.008\right)$; concretamente, en la de mujeres $\left(Z_{U}=-0.102, p=0.918 ; r=-0.005\right)$ y en la de hombres $\left(Z_{u}=-0.159, p=0.874 ; r=-0.008\right)$.

La edad presentó correlaciones significativas, bajas y negativas con la satisfacción sexual (ISS-10) en las tres muestras, con varianzas compartidas mayores que 4 \% y menores del 9 \% (Tabla 3). Además, en las tres muestras, estas correlaciones permanecieron significativas, bajas y negativas tras parcializar el engrandecimiento marital (MAS-14), con varianzas compartidas mayores que $5 \%$ y menores que $9 \%$, incrementándose ligeramente en la muestra total y en la de hombres. Tras parcializar la satisfacción con la relación (RAS), las correlaciones aumentaron y pasaron a ser moderadas, con varianzas compartidas mayores que $10 \%$ y menores que $11 \%$ (Tabla 3 ). Definidos cuatro grupos de edad (18-29, 30-39, 40-49 y $\geq 50$ ), se contrastó el efecto de interacción entre el sexo y la edad sobre el ISS-10 por la prueba de rangos alineados, y este no resultó significativo $(F[3,799]=0.153, p=0.928$; $\eta^{2}=0.001$ ). En la Tabla 3, se puede ver el cálculo de los deciles y cuartiles de los cuatro grupos de edad.

El tiempo que lleva la pareja casada o en unión libre presentó correlaciones significativas, bajas y negativas con la satisfacción sexual (ISS-10) en las tres muestras, con varianzas compartidas mayores que $7 \%$ y menores que $8 \%$. Tras parcializar el engrandecimiento marital (MAS-14), prácticamente no variaron las correlaciones, sino que disminuyeron muy ligeramente en la muestra total y en la de mujeres. No obstante, al parcializar la satisfacción con la relación (RAS), las correlaciones se incrementaron y pasaron a ser moderadas, resultando las varianzas compartidas mayores que $9 \%$ y menores que $11 \%$ (Tabla 3). Definidos dos grupos con base en la mediana (9 años), el efecto de interacción sexo-tiempo de casados/unión libre sobre el ISS-10 no fue significativo $\left(F[1,803]=0.068, p=0.795 ; \eta^{2}<0.001\right)$. En la tabla 3 se puede ver el cálculo de los deciles y cuartiles de los dos grupos de años de casados o en unión libre $(\leq 9$ y $>9)$. 
El número de hijos presentó correlaciones significativas, bajas y negativas con la satisfacción sexual (ISS-10) en las tres muestras, con varianzas compartidas mayores que $5 \%$ y menores que $7 \%$. Tras parcializar el engrandecimiento marital (MAS-14) o la satisfacción con la relación (RAS), estas correlaciones descendieron, pero siguieron siendo significativas, negativas y bajas (Tabla 3). Definidos cinco grupos (0 hijos, 1, 2, 3 y $\geq 4$ ), el efecto de interacción sexo-número de hijos no fue significativo $\left(F[4,797]=0.543, p=.704 ; \eta^{2}=\right.$ $0.003)$.

La escolaridad presentó correlaciones significativas, bajas y positivas con la satisfacción sexual (ISS-10) en las tres muestras, con varianzas compartidas mayores que $1 \%$ y menores que $2 \%$. Tras parcializar el engrandecimiento marital (MAS-14) o la satisfacción con la relación (RAS), estas correlaciones descendieron y dejaron de ser significativas en mujeres y hombres (Tabla 3). El efecto de interacción sexo-escolaridad sobre el ISS-10 no fue significativo $\left(F[4,797]=0.393, p=.814 ; \eta^{2}=0.002\right)$.

\section{DISCUSIÓN}

Un primer objetivo del estudio consistió en contrastar la invarianza del modelo de un factor (con 10 indicadores) entre ambos sexos, usando el método de modelos anidados con restricciones crecientes, es decir, imponiendo que la estimación del parámetro sea la misma en las dos muestras (Byrne, 2016). De acuerdo con la expectativa (Moral, 2009a), este modelo mostró buenas propiedades de invarianza con base en tres criterios: 1) la equivalencia de los parámetros de las cargas factoriales, varianza del factor y varianzas de los residuos entre ambos sexos (solapamiento de intervalos con una confianza del $95 \%$ entre las dos muestras), 2) los índices de buen ajuste a los datos en los cuatro modelos anidados y 3 ) la equivalencia de la bondad de ajuste entre los cuatro modelos por los índices diferenciales $\Delta \mathrm{GFI}, \Delta \mathrm{NFI}, \Delta \mathrm{CFI}$ y $\Delta \mathrm{RFI}$. Por tanto, el modelo es perfectamente válido para mujeres y hombres, lo que constituye de por sí una prueba de validez cruzada para el mismo.

Como segundo objetivo se propuso describir la distribución del ISS-10. Asumiendo que la expectativa de que las escalas que evalúan aspectos de satisfacción con la relación de pareja o familiar muestran sesgos hacia el polo de la satisfacción (Armenta-Hurtarte, Sánchez-Aragón \& Díaz-Loving, 2014; Caprara, Eisenberg \& Alessandri, 2016; Moral, 2009b), hubo mayor concentración de las puntuaciones en valores altos por encima de la media. Al dividir la puntuación total (con todos los ítems puntuados en sentido de satisfacción sexual) por el número de ítems sumados, se obtuvo una puntuación en el 
intervalo continuo de 1 a 7. De este modo, si se divide este continuo en siete intervalos (añadiendo medio punto a cada extremo como corrección de continuidad) y estos se disponen en sentido creciente en correspondencia con los siete valores discretos de respuesta a cada ítem, se pueden usar las etiquetas de respuesta para interpretar las puntuaciones dentro de cada intervalo, así: de 0.5 a $1.499=1$ = en ningún momento, de 1.5 a $2.499=$ $2=$ en muy raras ocasiones, de 2.5 a $3.499=3=$ algunas veces, de 3.5 a $4.499=4=$ parte del tiempo, de 4.5 a $5.499=5=$ buena parte del tiempo, de 5.5 a $6.499=6$ = la mayor parte del tiempo, y de 6.5 a $7.499=7=$ en todo momento. La media ( $M=5.33$, IC del $95 \%: 5.23,5.43$ ) quedó claramente incluida en el quinto intervalo, que corresponde a una respuesta de buena parte del tiempo, esto es, refleja satisfacción sexual. Si esta puntuación se transforma en índice y se puntúa hacia el polo de la insatisfacción: (1 - ([7 $-x] / 6)$ )*100), entonces el valor sería 72.16 (IC del $95 \%$ : 70.5, 73.83), lo que es una puntuación significativamente mayor que la media reportada por Moral (2009a), ya sea con los 25 ítems ( $M=60.86$; IC del $95 \%$ : 57.80, 63.92) o con los 10 ítems de su versión simplificada ( $M=63.19$; IC del $95 \%$ : 61.19, 65.67). Por tanto, la diferencia no puede atribuirse a la selección de los ítems más consistentes y que en su mayoría tienen una redacción en sentido afirmativo hacia la satisfacción sexual. Se puede afirmar más bien que la estimación de la media en la presente muestra es significativamente más alta. Debido a las características de la muestra (tamaño grande de 807 participantes, muestreo por rutas al azar y equiponderación en la secuencia de pruebas), la presente estimación posee más garantías de inferencia que el estudio de validación previo (muestra de 200 participantes, incidental y con una secuencia fija de pruebas).

El tercer objetivo del trabajo buscaba aportar pruebas de validez convergente en relación con la satisfacción con la relación y el engrandecimiento marital. Como en otros estudios (McNulty, Wenner \& Fisher; 2016; Sánchez et al., 2014), la correlación entre la satisfacción sexual y la satisfacción con la relación de pareja varió de alta a muy alta con una varianza compartida aproximada del 50 \% tanto en hombres como en mujeres; a su vez, la correlación entre la satisfacción sexual y el engrandecimiento marital resultó alta también en concordancia con estudios previos (Liu et al., 2017; Moral, 2011; Zhou et al., 2017), con una varianza compartida ligeramente mayor a un cuarto en ambos sexos.

Si se considera el engrandecimiento marital como un potencial sesgo distorsionador en el reporte de la satisfacción (Liu et al., 2017), se constata que 
la relación entre la satisfacción con la relación (RAS) y la satisfacción sexual (ISS-10) siguió siendo significativa y resultó alta, con más de un cuarto de la varianza compartida en ambos sexos, una vez que se parcializó la varianza del engrandecimiento marital (MAS); ello indica que se trata de una relación sustantiva. Asimismo, la relación entre la satisfacción sexual (ISS-10) y el engrandecimiento marital (MAS) fue sustantiva, una vez que se parcializó la varianza de la satisfacción con la relación (RAS). Por tanto, estos datos aportan evidencias adicionales de la validez de la escala.

Debe advertirse que Moral (2011) ofreció pruebas a favor de la interpretación de las puntuaciones en la MAS como un sesgo cognitivo-emocional relacionado con sentimientos de enamoramiento o amor ciego hacia la pareja, siendo distinto al sesgo distorsionar de la deseabilidad social (falsear el reporte en un sentido socialmente deseable). El sesgo evaluado por la MAS favorece el ajuste diádico y la duración de la pareja, y consecuentemente presenta una correlación alta en lo correspondiente a la satisfacción con la relación y satisfacción sexual, como revela este estudio. Concordante con esta interpretación, la relación sustantiva entre la satisfacción sexual y el engrandecimiento marital, una vez se elimina el efecto de la satisfacción con la relación de pareja, esto es, entre satisfacción sexual y la deseabilidad social, fue baja, tal como reportan las investigaciones que miden directamente dicho constructo (Muise et al., 2016; Moral, 2009b).

El cuarto objetivo del estudio fue comprobar el efecto de las variables sociodemográficas sobre las puntuaciones en el ISS-10. Se esperaba un tamaño de efecto trivial del sexo sobre la escala, con mayor media en los hombres que en las mujeres, al tratarse de una muestra de población general (Moral, 2009a; Sánchez et al., 2014). No obstante, el promedio de los hombres, aparentemente más alto, resultó estadísticamente equivalente al de las mujeres, teniendo el sexo un efecto trivial (no significativo) sobre la puntuación en el ISS-10, por lo que no se requirieron baremos diferenciales por sexo. Tampoco el hecho de estar casado o de vivir en unión libre tuvo efecto significativo sobre el ISS-10. A pesar de ello, la edad, el tiempo de casados o en unión libre y el número de hijos sí presentaron correlaciones bajas con el ISS-10 en mujeres y hombres, siendo todas estas correlaciones sustantivas ante el efecto de la satisfacción con la relación y el engrandecimiento marital (Fallis et al., 2016; Kettrey \& Carpenter, 2015; McNulty et al., 2016; Sánchez et al., 2014). Frente a estas tres variables con efecto decrementador sobre la satisfacción sexual, la escolaridad, aunque presentó un efecto incrementador, resultó espuria una vez se controló el efecto de la satisfacción con la relación 
y el engrandecimiento marital, lo que es concordante con el hecho de que en unos estudios resulte un correlato significativo y en otros, no (Kettrey \& Carpenter, 2015; Sánchez et al., 2014). Tomadas en conjunto, todas estas relaciones, conforme a las expectativas, aportan pruebas de validez adicionales para el ISS-10.

No se ha estudiado la validez de contenido del ISS, ni del ISS-10. Pero respecto a este tipo de validez, cabe citar el estudio de Pascoal, Narciso y Pereira (2014), autores portugueses que exploraron los significados de la satisfacción sexual en 810 personas heterosexuales con relaciones de pareja estables, encontrando dos dimensiones. Por una parte, aparecen los temas focalizados en aspectos positivos de la experiencia individual, como placer, emociones positivas, excitación, apertura y orgasmo; por otra parte, están los temas que enfatizan aspectos relacionales, como mutualidad, romance, expresión de sentimientos de amor, creatividad, externar deseo y frecuencia de actividad sexual. Los 10 ítems seleccionados para conformar el ISS-10 abarcan tantos aspectos relaciones (1. Creo que mi pareja disfruta de nuestra vida sexual, 3 . El sexo es divertido para mi pareja y para mí, 10 . Disfruto de las técnicas sexuales que mi pareja usa o le gustan, 17. Creo que el sexo es una función normal dentro de nuestra relación, 19. Creo que nuestra vida sexual realmente mejora nuestra relación, 22. Creo que mi pareja está satisfecha sexualmente conmigo, y 23. Mi pareja es muy sensible a mis necesidades y deseos sexuales) como personales (2. Nuestra vida sexual es muy excitante, 9. Mi pareja es sexualmente muy excitante, 12 . Creo que nuestra vida sexual es maravillosa, 16. Mi pareja es un/a amante maravilloso/a, y 21. Me es fácil excitarme sexualmente con mi pareja). No obstante, para establecer la validez de contenido se requeriría un panel de expertos y se podría trabajar con los criterios de Lawshe (Ayre \& Scally, 2014).

Como limitaciones del estudio, es de señalar que la técnica de rutas al azar, dentro de los procedimientos probabilísticos, es la que menos garantiza la aleatoriedad, especialmente si no se cuenta con un software que seleccione al azar $n$ rutas de todas las posibles (calles nominadas y enumeradas). Además, los datos solo pueden generalizarse a la población de la cual fueron extraídos (adultos heterosexuales casados o en unión libre de la ciudad de Monterrey, México). Para su aplicación en otras poblaciones, se requiere su validación. Al ser un estudio de corte transversal, su inferencia en la línea temporal (por ejemplo hacia el futuro) no está garantizada; además, no permite inferencias causales. Como fortaleza se destaca que la secuencia de presentación de las escalas, salvo la de depresión, se hizo mediante la técnica 
de equiponderación, que proporciona más garantías de distribución aleatoria de los posibles errores secuenciales. Esta técnica asume un error secuencial equivalente entre las pruebas, y para evitar un sesgo hacia la autodevaluación (Sui et al., 2016), se fijó la misma al final del cuestionario.

En conclusión, los datos proporcionaron evidencias de validez cruzada (entre mujeres y hombres) del modelo de un factor con 10 indicadores. Además, mostraron que el sexo no tiene ningún efecto sobre la distribución de las puntuaciones en el ISS-10, ya sea en una relación bivariada o en interacción con otras variables. Las puntuaciones del ISS-10 no siguieron una distribución normal, por lo que la escala puede ser baremada por puntuaciones de percentil, no requiriendo baremos diferenciales por sexo ni estado civil (casados y unión libre). Ahora bien, por el tamaño del efecto moderado de la edad y el tiempo de casados o en unión libre sobre el ISS-10, se podrían contemplar baremos diferenciales en relación con estas dos variables. En cambio, la asociación con el número de hijos fue sustantiva, pero con un tamaño del efecto pequeño; consecuentemente, no se requieren definir baremos diferenciales en relación con este aspecto. La mayor escolaridad tuvo un efecto incrementador; no obstante, su tamaño del efecto fue pequeño y resultó una variable espuria una vez se controló el efecto de la satisfacción con la relación y el engrandecimiento marital, por lo que tampoco se requieren baremos diferenciales en relación con la escolaridad. Los datos también proporcionaron evidencias de validez convergente para este modelo unidimensional de medida de la satisfacción sexual no solo por las relaciones esperadas con las variables sociodemográficas, sino también por las relaciones positivas y sustantivas en lo concerniente a la satisfacción con la relación y el engrandecimiento marital. Por las asociaciones altas con estas dos últimas variables, sería importante controlarlas al estudiar la relación del ISS-10 con otras variables. Precisamente, el sesgo puro de la deseabilidad social presente en el ISS-10 (correlación con engrandecimiento marital una vez parcializada la varianza de la satisfacción con la pareja) es pequeño, tal como reportan los estudios que miden directamente este constructo.

En general, se recomienda el uso del modelo unidimensional simplificado en la evaluación de la satisfacción sexual, pero se sugiere estudiar la fiabilidad temporal y la validez de contenido del ISS-10 y aportar nuevas evidencias de validez convergente con otras escalas, como las de comunicación en la pareja, actitudes hacia la sexualidad e imagen corporal. Otra aproximación a la validación del modelo unidimensional de la escala sería el modelo de Rasch y la teoría de la respuesta al ítem (Valdés, 2014). En este caso, los datos 
se podrían analizar mediante el programa WINSTEPS de Linacre (2017). En caso confirmatorio, estos análisis proporcionarían nuevas evidencias a favor de la unidimensionalidad del ISS-10.

\section{AGRADECIMIENTOS}

A la Dra. Melina Miaja Ávila por su apoyo en el trabajo de campo y captura de datos, mientras estuvo como becaria de investigación, y a los alumnos de Investigación en Psicología de primer semestre de la Facultad de Psicología de la Universidad Autónoma de Nuevo León, por la colecta de datos.

Nota de autores: Artículo derivado del proyecto de investigación: Infidelidad en el matrimonio: prevalencia, modalidades y factores de riesgo y protección.

\section{REFERENCIAS}

Ahumada, S., Lüttges, C., Molina, T. \& Torres, S. (2014). Satisfacción sexual: revisión de los factores individuales y de pareja relacionados. Revista Hospital Clínico Universidad de Chile; 25, 278-284. Recuperado de https://www.redclinica.cl/ Portals/0/Users/014/14/14/satisfaccion_sexual.pdf

Armenta-Hurtarte, C., Sánchez-Aragón, R. \& Díaz-Loving, R. (2014). Efectos de la cultura sobre las estrategias de mantenimiento y satisfacción marital. Acta de Investigación Psicológica, 4, 1572-1584. Recuperado de http://www.scielo.org. $\mathrm{mx} /$ scielo.php?script=sci_arttext\&pid=\$2007-48322014000200009

Asociación Americana de Psicología (APA). (2016). Ethical principles of psychologists and code of conduct ( $3^{\mathrm{a}} \mathrm{Ed}$.). Washington, DC: APA. Disponible en http://www. apa.org/ethics/code/

Ato, M., López, J. J. \& Benavente, A. (2013). Un sistema de clasificación de los diseños de investigación en psicología. Anales de Psicología, 29(3), 1038-1059. http:// dx.doi.org/10.6018/analesps.29.3.178511

Ayre, C. \& Scally, A. J. (2014). Critical values for Lawshe's content validity ratio: revisiting the original methods of calculation. Measurement and Evaluation in Counseling and Development, 47, 79-86. https://doi.org/10.1177/0748175613513808

Breakwell, G. M., Smith, J. A. \& Wright, D. B. (2012). Research methods in psychology. London, UK: Sage.

Byrne, B. M. (2016). Structural equation modeling with AMOS: Basic concepts, applications, and programming ( $3^{\mathrm{a}} \mathrm{Ed}$.). New York, NY: Routledge.

Caprara, G. V., Eisenberg, N. \& Alessandri, G. (2016). Positivity: The dispositional basis of happiness. Journal of Happiness Studies, 17, 1-19. https://doi.org/10.1007/ s10902-016-9728-y

Castellanos, E., Álvarez, C., Ruiz, D. \& Pérez, G. (2013). Social determinants of sexual satisfaction in Spain considered from the gender perspective. Annals of Epidemiology, 23, 150-156. https://doi.org/10.1016/j.annepidem.2012.12.010

Cheng, Z. \& Smyth, R. (2015). Sex and happiness. Journal of Economic Behavior \& Organization, 112, 26-32. https://doi.org/10.1016/j.jebo.2014.12.030 
Corder, G. W. \& Foreman, D. I. (2014). Nonparametric statistics: A step-by-step approach. USA: John Wiley \& Sons.

Daugherty, T. K., Julian, H. M., Lynch, N. M., Chen, S. J., Whipple, T. L. \& Ginsburg, A. F. (2016). Beyond the absence of disease or infirmity: the case for sexual wellness. College Student Journal, 50, 404-408. Recuperado de http://www.ingentaconnect.com/content/prin/csj/2017/00000050/00000003/art00012

Díaz, A. J., Bulla, Y. C. \& Moreno, J. (2016). Percepción de satisfacción sexual y su relación con estrés académico en estudiantes de odontología (Tesis doctoral). Universidad de Cartagena. Cartagena, Colombia. Disponible en http://190.242.62.234:8080/ jspui/handle/11227/4845

Fallis, E. E., Rehman, U. S., Woody, E. Z. \& Purdon, C. (2016). The longitudinal association of relationship satisfaction and sexual satisfaction in long-term relationships. Journal of Family Psychology, 30, 822-831. http://dx.doi. org/10.1037/fam0000205

García, J. A., Reding, A. \& López, J. C. (2013). Cálculo del tamaño de la muestra en investigación en educación médica. Investigación en Educación Médica, 2(8), 217-224. https://doi.org/10.1016/S2007-5057(13)72715-7

Hendrick, S. S. (1988). A generic measure of relationship satisfaction. Journal of Marriage and the Family, 50, 93-98. https://doi.org/10.2307/352430

Hudson, W. W. (1992). The WALMYR assessment scales scoring manual. Tallahassee, FL: WALMYR Publishing Co.

Instituto Nacional de Estadística, Geografía e Informática (2015). Panorama sociodemográfico de Nuevo León. Ciudad de México: INEGI. Disponible en http://internet. contenidos.inegi.org.mx/contenidos/Productos/prod_serv/contenidos/espanol/ bvinegi/productos/nueva_estruc/inter_censal/panorama/702825082291.pdf

Kerby, D. S. (2014). The simple difference formula: An approach to teaching nonparametric correlation. Comprehensive Psychology, 3(1), 1-9. https://doi. org/10.2466/11.IT.3.1

Kettrey, H. H. \& Carpenter, L. M. (2015). Satisfaction, sexual. En P. Whelehan \& A. Bolin (Eds.), The international encyclopedia of human sexuality, 3, 1115-1354. https://doi.org/10.1002/9781118896877.wbiehs422

Kismödi, E., Cottingham, J., Gruskin, S. \& Miller, A. M. (2015). Advancing sexual health through human rights: The role of the law. Global Public Health, 10, 252-267. https://doi.org/10.1080/17441692.2014.986175

Leavitt, C. E., McDaniel, B. T., Maas, M. K. \& Feinberg, M. E. (2016). Parenting stress and sexual satisfaction among first-time parents: A dyadic approach. Sex Roles, 76, 346-355. https://doi.org/10.1007/s11199-016-0623-0

Linacre, J. M. (2017). Winsteps (Version 4.0.1) [Computer Software]. Beaverton OR: Winsteps.com.

Liu, J., Wang, Y. \& Jackson, T. (2017). Towards explaining relationship dissatisfaction in Chinese dating couples: Relationship disillusionment, emergent distress, or insecure attachment style? Personality and Individual Differences, 112, 42-48. https://doi.org/10.1016/j.paid.2017.02.041

Mark, K. P., Herbenick, D., Fortenberry, J. D., Sanders, S. \& Reece, M. (2014). A psychometric comparison of three scales and a single-item measure to assess 
sexual satisfaction. The Journal of Sex Research, 51, 159-169. https://doi.org/10. 1080/00224499.2013.816261

McNulty, J. K., Wenner, C. A. \& Fisher, T. D. (2016). Longitudinal associations among relationship satisfaction, sexual satisfaction, and frequency of sex in early marriage. Archives of Sexual Behavior, 45, 85-97. https://doi.org/10.1007/ s10508-014-0444-6

Moral, J. (2009a). Medida de la satisfacción sexual en parejas casadas: un estudio de validación. Archivos Hispanoamericanos de Sexología, 15(1), 46-70.

Moral, J. (2009b). Control de la deseabilidad social en el autorreporte del ajuste diádico y satisfacción marital. Psicología y Ciencia Social, 9(2), 5-12. Disponible en http://cuved.unam.mx/ojs/index.php/pycs/article/view/167

Moral, J. (2011). Predicción de la satisfacción sexual en mujeres y hombres casados. Revista Mexicana de Investigación en Psicología, 3(1), 85-102. Disponible en http://new.medigraphic.com/cgi-bin/resumen.cgi?IDARTICULO=44493

Moral, J. (2015). Validación del modelo unidimensional de la Escala de Valoración de la Relación en personas casadas y en unión libre de Monterrey, México. Revista Internacional de Psicología, 14(2), 1-70. Disponible en http://www.revistapsicologia.org/index.php/revista/article/view/134

Moral, J. (2016). Invarianza factorial de la escala de engrandecimiento marital por sexo. Revista Informes Psicológicos, 16(1), 117-135. http://dx.doi. org/10.18566/10.18566/infpsicv16n1a07

Muise, A., Stanton, S. C. E., Kim, J. J. \& Impett, E. A. (2016). Not in the mood? Men under- (not over-) perceive their partner's sexual desire in established intimate relationships. Journal of Personality and Social Psychology, 110, 725-742. https:// doi.org/10.1037/pspi0000046

Oliver, J. C., González, J. \& Rosel, J. (2014). Análisis no paramétrico de la interacción de dos factores mediante el contraste de rangos alineados. Psicothema, 21, 152-158. Disponible en http://www.psicothema.com/psicothema.asp?id=3608

O'Rourke, N. \& Cappeliez, P. (2002). Development and validation of a couples measure of biased responding: The Marital Aggrandizement Scale. Journal of Personality Assessment, 78, 301-320. https://doi.org/10.1207/S15327752JPA7802_06

Pascoal, P. M., Narciso, I. D. S. B. \& Pereira, N. M. (2014). What is sexual satisfaction? Thematic analysis of lay people's definitions. Journal of Sex Research, 51, 22-30. https://doi.org/10.1080/00224499.2013.815149

Sánchez, M. del M., Santos, P. \& Sierra, J. C. (2014). A systematic review of sexual satisfaction. International Journal of Clinical and Health Psychology, 14, 67-75. https://doi.org/10.1016/S1697-2600(14)70038-9

Secretaría de Salud (4 de enero, 2013). NORMA Oficial Mexicana NOM-012-SSA3-2012, Que establece los criterios para la ejecución de proyectos de investigación para la salud en seres humanos. Diario Oficial de la Federación. Recuperado de http:// dof.gob.mx/nota_detalle.php?codigo $=5284148 \&$ fecha $=04 / 01 / 2013$

Sociedad Mexicana de Psicología (2007). Código ético del psicólogo (4ª ed.). Ciudad de México: Trillas.

Sui, J., Ohrling, E. \& Humphreys, G. W. (2016). Negative mood disrupts self-and reward-biases in perceptual matching. The Quarterly Journal of Experimental Psychology, 69, 1438-1448. https://doi.org/10.1080/17470218.2015.1122069 
Valdés, H. (2014). Introducción a la teoría de respuestas al ítem. TRI. Santo Domingo, República Dominicana: Ministerio de Educación e Instituto Dominicano de Evaluación e Investigación de la Calidad Educativa. Recuperado de https://www. uam.es/personal_pdi/psicologia/cadalso/Docencia/PoliTRI/TRI4_v2.pdf

Veit, M., Štulhofer, A. \& Hald, G. M. (2016). Sexually explicit media use and relationship satisfaction: a moderating role of emotional intimacy? Sexual and Relationship Therapy, 32(1), 1-17. https://doi.org/10.1080/14681994.2016.1193134

Zhou, R. D., Chiu, M. Y. \& Chui, W. Y. (2017). Development and validation of the Marital Metaphor Questionnaire (MMQ-10) for urban Chinese women. Journal of Marital and Family Therapy, 43, 65-81. https://doi.org/10.1111/jmft.12181 autor o el licenciante.

\section{(cc) BY}

